\section{Caffeine intake is associated with pupil dilation and enhanced accommodation}

\begin{abstract}
Purpose It is purported that caffeine, an autonomic stimulant, affects visual performance. This study sought to assess whether caffeine intake was associated with changes in pupil size and/or amplitude of accommodation.
\end{abstract}

Patients and methods A double-masked, crossover study was conducted in $\mathbf{5 0}$ healthy subjects of age range 19 to 25 years.

Subjects were randomized to treatments such that subjects consumed either $250 \mathrm{mg}$ caffeine drink or vehicle on separate days. Amplitude of accommodation was measured by the push-up technique, and pupil size using a millimeter ruler fixed to a slit lamp biomicroscope in dim illumination (5 lux). Amplitude of accommodation and pupil size were taken at baseline, and at 30,60 and 90 min time points post treatment. Repeated measures one-way ANOVA and paired $t$-test were used in analyzing data.

Results Amplitude of accommodation and pupil size after caffeine intake were significantly greater than vehicle $(P<0.001)$ at each time point. Consumption of the caffeine beverage was associated with significant increases in amplitude of accommodation and pupil size with time $(P<0.001)$. Amplitude of accommodation rose from $12.4( \pm 2.2 \mathrm{D})$ at baseline to 15.8 $( \pm 2.6 \mathrm{D})$ at $90 \mathrm{~min}$. Similarly, pupil size increased from $3.4( \pm 0.4 \mathrm{~mm})$ at baseline to $4.5( \pm 0.72 \mathrm{~mm})$ at $90 \mathrm{~min}$. Consumption of vehicle was not associated with increase in amplitude of accommodation or pupil size with time.

Conclusion Pupil size and accommodation are affected after ingestion of caffeine.

This study suggests caffeine may have some influence on visual functions.

Eye (2017) 31, 615-619; doi:10.1038/eye.2016.288; published online 16 December 2016

\section{Introduction}

Pupillary response and accommodation are important physiological processes of the eye that affect vision. Ocular accommodation is a blurdriven reflex and results in focusing of images onto the retina. ${ }^{1}$ The pupil regulates retinal illumination to optimize visual perception. Other visual functions such as contrast sensitivity, color perception, and visual acuity are influenced by pupil size and accommodation. ${ }^{2}$ The iris and ciliary muscles, which are directly responsible for the manifest structural changes in pupillary response and accommodation respectively, receive autonomic innervations from both the mesencephalic Edinger-Westphal nucleus and hypothalamic center in the diencephalon. ${ }^{3-5}$ Parasympathetic innervation of the iris sphincter muscle results in reduction of pupillary diameter, whereas sympathetic innervation of the iris dilator muscle increases pupil diameter. However, the accommodative state is largely controlled by parasympathetic innervation of the ciliary muscle, although sympathetic innervation plays a complementary role in relaxing accommodation. ${ }^{6}$

Caffeine, a psychoactive drug, is commonly consumed in various forms and in variable amounts by people worldwide. The absorption of caffeine into the bloodstream after oral intake is very efficient, and widely distributed to all organs of the body including the nervous system because of its hydrophobic properties. 7,8 Interestingly, caffeine has wide variations in its nature of action on biological tissues, ${ }^{9}$ an attribute that makes it worth of study.

Because of the increased awareness of the wide spread effects of caffeine on the nervous system, ${ }^{8}$ scientists have sought to investigate for ocular and visual changes associated with caffeine intake. This is because ocular tissues including the iris muscles, ciliary muscle,
${ }^{1}$ Department of Optometry, School of Allied Health Sciences, College of Health and Allied Sciences, University of Cape Coast, Cape Coast, Ghana

${ }^{2}$ School of Optometry, Hong Kong Polytechnic University, Hong Kong

${ }^{3}$ School of Optometry, University of Alabama at Birmingham, Birmingham, AL, USA

Correspondence: S Abokyi, School of Optometry, Hong Kong Polytechnic University, Kowloon, Hong Kong Tel: +852 51086048 . E-mail: samyomah22@ yahoo.com or sabokyi@ucc. edu.gh

Received: 19 September 2016

Accepted: 8 November 2016

Published online:

16 December 2016 
smooth muscles of the eyelids, lacrimal gland, and choroidal and conjunctival blood vessels receive autonomic innervation. ${ }^{10}$ Although results of studies may be inconsistent, caffeine intake has been associated with changes in ocular parameters including tear secretion, intraocular pressure, and macular perfusion. ${ }^{11-13}$ Recently, some researchers have found significant changes in some vision-related tasks associated with caffeine intake. ${ }^{14,15}$ We therefore hypothesized that caffeine could affect visual performance through changes in pupil size and/or accommodation.

\section{Materials and methods}

\section{Ethical consideration}

Ethical approval was obtained from the Institutional Review Board of the University of Cape Coast. The research protocol followed the tenets of the Declaration of Helsinki for use of human subjects, and informed consent was obtained from all subjects before participation.

\section{Sampling procedure}

Fifty healthy subjects of either sex, aged 19 to 25 years and weighing 59 to $65 \mathrm{~kg}$, were sampled from the student population of the university. Subjects underwent a preliminary eye examination before recruitment. The eligibility criteria were refractive correction of spherical equivalent $\leq \pm 0.50 \mathrm{D}$ and best spectacle corrected visual acuity of $6 / 6$ or better. Persons excluded were those having systemic or ocular health diseases,

accommodative anomalies or pupillary defects, as well as history of habitual ingestion of caffeine-containing beverages in order to eliminate tolerance effects due to chronic use. Subjects were not supposed to have consumed any caffeine at least a week before the study.

\section{Experimental protocol}

A double-blind, placebo-controlled, randomized crossover experimental design was conducted. Subjects were allowed to sit in the examination room for $15 \mathrm{~min}$ after which baseline measurements for pupil size and accommodation were taken. The value recorded for pupil size and amplitude of accommodation was the average of three closest measures out of five taken per subject.

Subjects were then randomized to treatments on separate days such that each received either $250 \mathrm{mg}$ caffeine (NuSci Caffeine Anhydrous Pure Powder, HerbStore, Walnut, CA, USA) dissolved in a $250 \mathrm{ml}$ lemon drink or same volume of placebo with reversal of the treatment on the second visit. This was done to ensure examiners were masked to the treatments to eliminate any potential examiner bias. The second treatment was received a week after the first as this was necessary for the washout phase of caffeine. Pupil size and amplitude of accommodation were measured at 30,60, and 90 min post treatment. All measurements were taken on the same eye that was randomly selected per subject at the start of the study. Two masked examiners took all measurements, one measured amplitude of accommodation and the other pupil diameter.

\section{Measurement of subjective amplitude of accommodation}

The monocular amplitude of accommodation was measured through distance spectacle correction. Spectacles were worn at $15 \mathrm{~mm}$ vertex distance. The subject's attention was directed to the 6/9 row of letters on a movable target of the Royal Air Force (RAF) ruler at $40 \mathrm{~cm}$ and instructed to keep the letters clear. Target was moved slowly at $1 \mathrm{~cm} / \mathrm{s}$ toward the nose and subjects were to report when the letters become and remain blurred. The distance from the target to spectacle plane was measured in $\mathrm{cm}$ and referred to as the near point of accommodation. The linear distance was converted into diopters by dividing the near point of accommodation in $\mathrm{cm}$ into 100. This represents the subject's accommodative amplitude.

\section{Pupil size}

Pupil size was measured using a ruler under a slit lamp microscope in a dimly lighted room (5 lux) with the face illuminated from below and pupils in a semidilated state as recommended for pupillary measurement. The subjects were made to fixate a distant target (6/9 letter of the Snellen chart). To measure pupil size, the ruler was positioned horizontally such that the zero calibration on the rule laid tangential and vertical on the temporal margin of the pupil. The horizontal pupil diameter was read off the vertical calibration that laid tangential to the inner margin of the pupil.

\section{Repeatability of measurements}

The two masked examiners, who are experienced clinicians, measured amplitude of accommodation or pupil size of subjects, using the push-up and mm ruler respectively, on two separate occasions. Repeatability of each measurement (pupil size and accommodation) taken per examiner was evaluated using intraclass correlation coefficients (ICCs) and 95\% limits of agreement (LoAs) to set the limits within which any change detected could be due to the repeatability of the measurement. The ICC and LoA for each subjective measurement were recorded 
(pupil size: $+0.91, \pm 0.6 \mathrm{~mm}$; amplitude of accommodation: $+0.82, \pm 1.4 \mathrm{D}$, respectively).

\section{Data analysis}

Statistical analysis of the data was done using Graphpad prism (GraphPad Software Inc., San Diego, CA, USA). Data was presented as mean $( \pm S D)$. The independent $t$-test was used to compare the amplitude of accommodation and pupil size between males and females. Repeated measures one-way ANOVA was used in evaluating the effect of time for each treatment. Paired $t$-test was used to compare amplitude of accommodation and pupil size between caffeine treatment and placebo treatment at specific time points. The threshold for statistical significance was set at a probability of $5 \%$.

\section{Results}

Fifty healthy subjects ( 29 males and 21 females) of mean age $22.2( \pm 1.9)$ years and weighing 59 to $65 \mathrm{~kg}$ (mean \pm SD: $62.7 \pm 2.4 \mathrm{~kg}$ ) participated in the study. At baseline, amplitude of accommodation and pupil size were $12.4 \pm 2.2 \mathrm{D}$ (range: $8.3-20.0 \mathrm{D}$ ) and $3.4 \pm 0.4 \mathrm{~mm}$ (range: $2.7-4.1 \mathrm{~mm}$ ) respectively. Amplitude of accommodation and pupil size did not differ significantly between males and females $(P>0.05)$.

Consumption of the caffeine-containing beverage by subjects resulted in increase in amplitude of accommodation and pupil diameter. Amplitude of accommodation rose from $12.4( \pm 2.2 \mathrm{D})$ at baseline to 15.8 $( \pm 2.6 \mathrm{D})$ at $90 \mathrm{~min}$. Similarly, pupil diameter increased from $3.4( \pm 0.4 \mathrm{~mm})$ at baseline to $4.5( \pm 0.72 \mathrm{~mm})$ at $90 \mathrm{~min}$. Amplitude of accommodation increased in each subject at $30 \mathrm{~min}$ post treatment with caffeine except in 3 male subjects in whom a decline was found (difference: $-1.1,-1.79$, and -1.8 D). Repeated measures one-way ANOVA showed significant increase in amplitude of accommodation $(\mathrm{F}(3,199)=100, P<0.001)$ and pupil size $(\mathrm{F}(3,199)=160, P<0.001)$ with time after subjects consumed caffeine. The post hoc analysis using the Bonferroni's multiple comparison test showed that amplitude of accommodation and pupil size were significantly greater at all specific time points post treatment with caffeine than baseline. Comparisons between consecutive time points post treatment indicated that amplitude of accommodation and pupil size increased significantly $(P<0.05)$ only at 30 and $60 \mathrm{~min}$. For the placebo treatment, however, variations in either amplitude of accommodation or pupil size with time in the subjects were not significant (repeated measures oneway ANOVA; $P>0.05)$. In addition, comparisons between the two treatments using paired $t$-test at each time point showed that the amplitude of accommodation
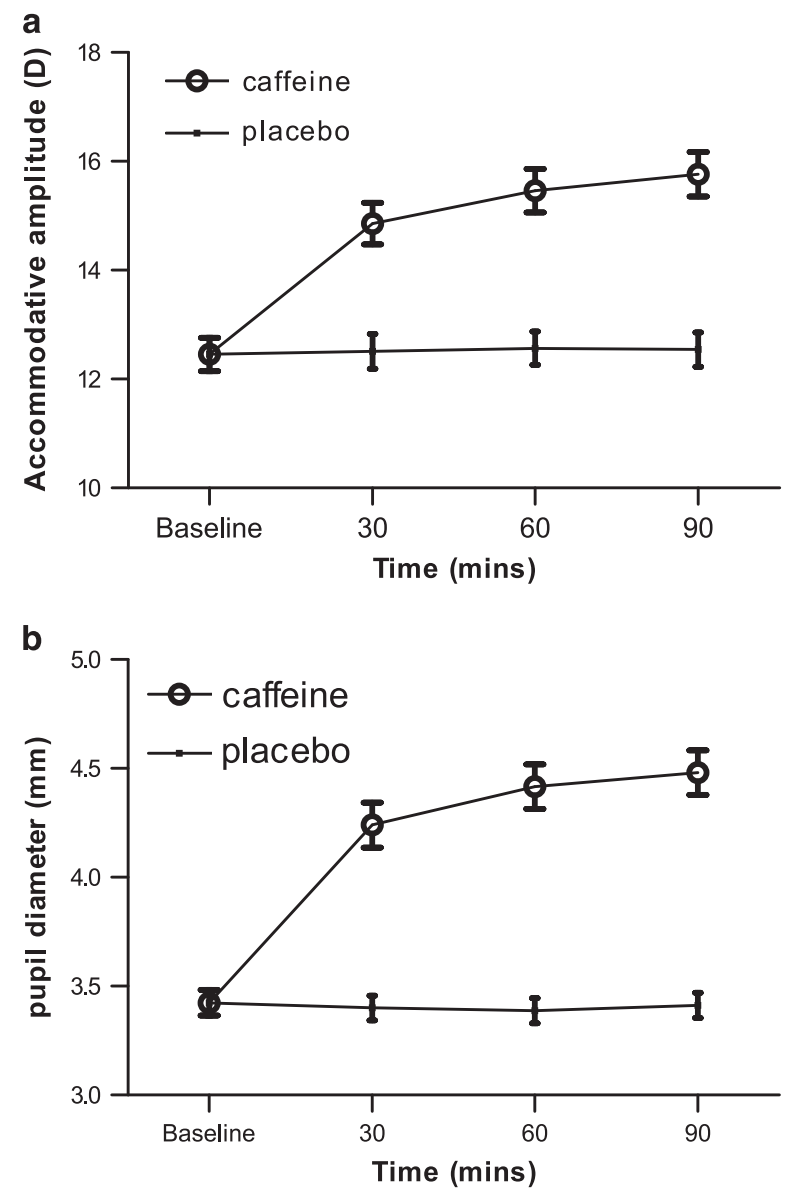

Figure 1 (a) Time courses of subjects' amplitude of accommodation at baseline and at 30,60, and 90 min post treatment with $250 \mathrm{mg}$ caffeine or placebo. (b) Time courses of subjects' pupil size at baseline and at 30,60, and 90 min post treatment with $250 \mathrm{mg}$ caffeine or placebo.

and pupil size were significantly higher in subjects after treatment with caffeine than with placebo (Figures 1a and b).

\section{Discussion}

Caffeine belongs to a class of weak stimulants called xanthines. Its supposedly beneficial effects perhaps influence its wide use. We found that amplitude of accommodation increased and pupil diameter enlarged significantly $(P<0.001)$ after the ingestion of caffeinecontaining beverage compared with vehicle. The choice of crossover experimental design, whereby same subjects received placebo and caffeine treatments, was to eliminate the intersubject variability that comes with the usual experimental-control group design. The results of this study are in agreement with findings of earlier studies by other researchers suggesting changes of accommodative functions after caffeine consumption. ${ }^{15-17}$ Kirshner and 
Schmid ${ }^{15}$ investigated the extent to which the eye is able to relax accommodation through a $+4.00 \mathrm{D}$ lens after consumption of $150 \mathrm{mg}$ caffeine in 11 subjects. The conclusion was that caffeine increased the tonicity of the ciliary muscles by about half of a diopter, hence resulting in the rejection of reading plus lens. In addition, Zhai et $a l^{16}$ studied changes in accommodative response at varied accommodative stimuli levels in 17 subjects before and after consumption of caffeine. They reported that caffeine increased the accommodative response only when the accommodative stimulus (object vergence) was not $<5.00 \mathrm{D}$. We measured amplitude of accommodation of younger subjects with greater accommodation ability, hence all our subjects had a sustained blur at a distance of $<20 \mathrm{~cm}(5.00 \mathrm{D})$. An increase of 2.2 D in amplitude of accommodation was observed at $30 \mathrm{~min}$ after caffeine intake. A similar increase in amplitude of accommodation of $\sim 2.00 \mathrm{D}$ was observed in another study by Ajayi and George ${ }^{17}$ in healthy Nigerian subjects who had consumed caffeinated coffee. It was found that pupil size and amplitude of accommodation increased with time after caffeine intake; a significant increase was observed first at $30 \mathrm{~min}$ and further increase at $60 \mathrm{~min}$. This observation might be related to the bioavailability of the drug in our subjects. According to a study by Blanchard and Sawers, ${ }^{18}$ it takes averagely $40 \mathrm{~min}$ for orally administered caffeine to reach its peak plasma concentration, and that it takes not less than $3 \mathrm{~h}$ for it to wear off completely.

If caffeine improves accommodation, alertness, and concentration, ${ }^{19}$ which are crucial for effective reading, why then did Kirshner and Schmid ${ }^{15}$ observe a negative correlation between caffeine intake and reading improvement, and furthermore, an improvement in reading ability in subjects upon reduction in caffeine intake? Our observation of an enlargement in pupil size after caffeine consumption, although measured in the passive state, provides some clue for the poor performance in reading by subjects after consuming caffeine. An enlarged pupil is associated with increased spherical aberration. ${ }^{20}$ This is because peripheral and central light rays pass through the pupil and are differently focused after refraction resulting in deterioration in vision.

An inference that caffeine enhances both parasympathetic and sympathetic nerve activity could be made from this study as it increased amplitude of accommodation and dilated the pupils. Reports on the effect of caffeine on autonomic nervous activity are inconsistent. Whereas some studies favor an enhancement of sympathetic nerve activity, ${ }^{21}$ others support enhanced parasympathetic nerve activity. ${ }^{22}$ Our results, however, corroborate an overall enhancement of autonomic innervation to the intrinsic muscles of the eye.
Enhancement of the parasympathetic nerve activity could account for contraction of the ciliary body and relaxation of the zonules, resulting in increased convexity of the crystalline lens (ie, accommodation), whereas increased sympathetic nerve activity results in contraction of the pupil dilator muscle causing pupil dilation. ${ }^{23}$ The mechanism behind the parasympathetic stimulation by caffeine is that it competitively binds to adenosine receptors, ${ }^{23}$ resulting in the rise in levels of acetylcholine that stimulates the muscarinic receptors of the ciliary body leading to its contraction. Caffeine also inhibits the actions of adenosine, responsible for modulation of other neurotransmitters. Consequently, there is rise in the levels of neurotransmitters including epinephrine and norepinephrine, or adrenaline, leading to activation of the sympathetic nerve activity.

According to the outcome of this study, caffeine has paradoxical effects on the intrinsic muscles of the eye: dilating the pupils and increasing accommodative amplitude. This may account for the changes in some visual functions or vision-related task associated with caffeine intake.

A notable limitation of this study is that it is not possible to infer whether caffeine intake could result in improvement or deterioration of near or distant vision. This is because the final retinal image formed does not only depend on the interplay between pupil size and accommodative status. Hence, we would suggest further investigation in this area. In addition, the subjects recruited into this study were healthy young adults and who were not habitual consumers of caffeine of any form, and hence the findings of this research may not be applicable to older adults or habitual consumers of caffeine. Also, we would suggest that further studies be conducted to determine the onset, peak, and maximum duration of caffeine on the amplitude of accommodation and pupil size.

\section{Summary}

What was known before

- It is purported that caffeine, an autonomic stimulant, affects visual performance.

What this study adds

- This study evaluates this assertion by investigating the effect of caffeine on pupil size and amplitude of accommodation. The outcome of this study provides the possible mechanism by which caffeine intake could affect vision.

\section{Conflict of interest}

The authors declare no conflict of interest. 


\section{Author contributions}

The study was conceived and designed by SA; JO-M was involved in the conduct of data collection; SA and JO-M carried out statistical analysis; SA drafted initial manuscript; KAO reviewed the content of the manuscript for publication. All authors read and approved the final manuscript.

\section{References}

1 Phillips S, Stark L. Blur: a sufficient accommodative stimulus. Doc Ophthalmol 1977; 43(1): 65-89.

2 Alfonso JF, Fernández-Vega L, Baamonde MB, Montés-Micó R. Correlation of pupil size with visual acuity and contrast sensitivity after implantation of an apodized diffractive intraocular lens. J Cataract Refract Surg 2007; 33(3): 430-438.

3 Loewenfeld IE. The Pupil: Anatomy, Physiology, and Clinical Applications. Iowa State University Press State University Press: Ames, IA; Detroit, MI, 1993, p 1594.

4 Cogan DG. Accommodation and the autonomic nervous system. Arch Ophthalmol 1937; 18: 739-766.

5 Meesmann A. Experimentelle untersuchungen uber die antagonistische innervation der ciliarmuskulatur. Albrecht von Graefes Arch Ophthalmol 1952; 152: 335-356.

6 Gilmartin B, Mallen EA, Wolffsohn JS. Sympathetic control of accommodation: evidence for inter-subject variation. Ophthalmic Physiol Opt 2002; 22(5): 366-371.

7 Marks V, Kelly JF. Absorption of caffeine from tea, coffee, and coca cola. Lancet 1973; 1(7807): 827.

8 Nehlig A, Daval JL, Debry G. Caffeine and the central nervous system: mechanisms of action, biochemical, metabolic and psychostimulant effects. Brain Res Brain Res Rev 1992; 17(2): 139-170.

9 Remington LE. Clinical Anatomy of the Visual System, 2nd ed. Elsevier Inc. Butterworth Heinemann: Philadelphia, PA, USA, 2005, p 254.

10 Leonard TK, Watson RR, Mohs ME. The effects of caffeine on various body systems: a review. J Am Diet Assoc 1987; 87(8): 1048-1053.
11 Osei KA, Ovenseri-Ogbomo G, Kyei S, Ntodie M. The effect of caffeine on tear secretion. Optom Vis Sci 2014; 91(2): 171-177.

12 Jiwani AZ, Rhee DJ, Brauner SC, Gardiner MF, Chen TC, Shen LQ et al. Effects of caffeinated coffee consumption on intraocular pressure, ocular perfusion pressure, and ocular pulse amplitude: a randomized controlled trial. Eye 2012; 26(8): 1122-1130.

13 Okuno T, Sugiyama T, Tominaga M, Kojima S, Ikeda T. Effects of caffeine on microcirculation of the human ocular fundus. Jpn J Ophthalmol 2002; 46(2): 170-176.

14 Coren S. The effect of caffeine ingestion on the perceived instability of visual patterns. Int J Psychophysiol 2002; 43(2): 185-189.

15 Kirshner AJ, Schmid R. The effects of caffeine on near point plus acceptance. J Am Optom Assoc 1984; 55(2): 97-102.

16 Zhai H, Goss DA, Hammond RW. The effect of caffeine on the accommodative response/accommodative stimulus function and on the response AC/A ratio. Curr Eye Res 1993; 12(6): 489-499.

17 Ajayi O, George O. Acute effect of caffeine on amplitude of accommodation and near point of convergence. West Afr J Pharmacol Drug Res 2008; 22(1): 27-30.

18 Blanchard J, Sawers SJA. The absolute bioavailability of caffeine in man. Eur J Clin Pharmacol 1983; 24(1): 93-98.

19 Glade MJ. Caffeine- not just a stimulant. Nutrition 2010; 26(10): 932-938.

20 McKelvie J, McArdle B, McGhee C. The influence of tilt, decentration, and pupil size on the higher-order aberration profile of aspheric intraocular lenses. Ophthalmology 2011; 118(9): 1724-1731.

21 Corti R, Binggeli C, Sudano I, Spieker L, Hänseler E, Ruschitzka $\mathrm{F}$ et al. Coffee acutely increases sympathetic nerve activity and blood pressure independently of caffeine content Role of habitual versus non habitual drinking. Circulation 2002; 106: 2935-2940.

22 Hibino G, Moritani T, Kawada T, Fushiki T. Caffeine enhances modulation of parasympathetic nerve activity in humans: quantification using power spectral analysis. J Nutr 1997; 127: 1422-1427.

23 Kardon R Anatomy and physiology of the autonomic nervous system. In: Miller NR, Newman NJ, Biousse V, Kerrison JB (eds). Walsh and Hoyt Clinical Neuro-Ophthalmology, $6^{\text {th }}$ ed. Williams \& Wilkins: Baltimore, 2005, p 649-711. 